\title{
Bagging und Air Stacking: Ein atemtherapeutischer Ansatz für Patienten in der neurologischen Frührehabilitation*
}

\author{
Respiratory Therapy (Bagging, Air Stacking) for Patients in Early \\ Neurorehabilitation
}

Autoren

Institute

\section{K. Frank $k^{1}$, U. Frank ${ }^{2}$}

Aatalklinik Wünnenberg, Klinik für neurologische und neurochirurgische Rehabilitation, Zentrum für interdisziplinäre Frührehabilitation (Klinikleitung: Prof. Dr. W. Tackmann)

Department Linguistik, Lehrstuhl für kognitive Neurolinguistik/Patholinguistik Universität Potsdam eingereicht 4.10.2010 akzeptiert nach Revision 10.1.2011

\section{Bibliografie}

DOI http://dx.doi.org/ 10.1055/s-0030-1256181

Online-Publikation: 10.2.2011

Pneumologie 2011; 65:

314-319 ๔ Georg Thieme

Verlag KG Stuttgart · New York ISSN 0934-8387

\section{Korrespondenzadresse} Katrin Frank

Aatalklinik Wünnenberg In den Erlen 22

33181 Bad Wünnenberg katrin.frank@freenet.de

\section{Zusammenfassung \\ $\nabla$}

In einem Pilotprojekt wurde untersucht, ob eine adaptierte atemtherapeutische Methode (Bagging) bei Patienten der Frührehabilitation zu verbesserten $\mathrm{SPO}_{2}$ Werten führt. Beim Bagging wird während der Inspirationsphase mit Hilfe eines Beatmungsbeutels zusätzlich Luft in die Lunge gegeben. Der Patient erhält nachfolgend eine manuelle Hustenunterstützung. Es wurden 11 Probanden untersucht, diese erhielten in einem Behandlungszeitraum von 12 Tagen 1-2× täglich eine Bagging-Anwendung. Es wurden Gruppen- und Einzelfallanalysen gemäß einer Zuordnung in $\mathrm{SPO}_{2}$-Kategorien durchgeführt (Kat. 1: $\leq 90 \%$, Kat. 2: $\leq 94 \%$, Kat. 3: > 94\%). Die Bagging-Therapie führte bei allen Probanden zu einer stabilen Erhöhung der $\mathrm{SPO}_{2}$-Werte. Alle Patienten konnten sich im Behandlungsverlauf um mindestens eine Kategorie verbessern. Ein Zusammenhang zwischen Therapieverlauf und Diagnose der einzelnen Patienten bestand nicht. Weitere positive Effekte waren eine Verbesserung der Vigilanz, der Bronchialsekretviskosität und der Schluckfunktion. Die Methode ist leicht zu erlernen und die Materialkosten sind gering, sodass sie gut in klinischtherapeutische Abläufe zu integrieren ist.

\section{Einleitung}

$\nabla$

Das Aufgabengebiet des Physiotherapeuten in der neurologischen Frührehabilitation ist so vielfältig wie die unterschiedlichen neurologischen Störungsbilder. Das Aufgabenspektrum hat sich zudem, u.a. durch gestiegene Überlebensraten von Patienten mit schwersten Hirnverletzungen [1], stark gewandelt. Viele Patienten sind den Frühre-

\footnotetext{
* Ausgewählte Inhalte dieser Arbeit wurden bei der Jahrestagung der Deutschen Gesellschaft für Neurorehabilitation (DGNR) und der Deutschen Gesellschaft für Neurotraumatologie und klinische Neurorehabilitation (DGNKN) am 3.-5.12. 2009 in Berlin präsentiert.
}

\section{Abstract \\ $\nabla$}

In a pilot project we examined whether an improvement of oxygen saturation $\left(\mathrm{SPO}_{2}\right)$ can be established by applying a modified respiratory intervention technique (bagging) for patients in early neurorehabilitation. The "bagging" method involves adding air into the lungs during the inspiration phase by using a resuscitation bag. Subsequently, the patient is requested to cough, and is given manual support on the chest by the therapist. We examined a group of 11 patients who received 1-2 bagging interventions per day over a period of 12 days. By applying the bagging method, a continuous and stable improvement of oxygen saturation could be accomplished for all patients. A single case analysis confirmed this effect for each patient. Furthermore, we observed an improvement of the patients' vigilance, improved quality of bronchial secretion and marked improvements in swallowing and phonation. The bagging method is an easy-to-learn and inexpensive method that leads to a stable and sustained increase in oxygen saturation and to improvement of other clinical parameters.

habilitationsphasen „B“ und „C“ zuzuordnen [2]. Die Schädigungsmuster sind häufig Kombinationen aus Vigilanzminderungen und erheblichen vegetativen Entgleisungen, dazu kommen motorische und kognitive Einschränkungen. Viele Patienten der Rehabilitationsphase B sind mit einer geblockten Trachealkanüle versorgt und haben eine unzureichende Sekretclearance. Hinzu kommen häufig Speichel- und Sekretaspirationen, z.B. als Folge einer Dysphagie. Eine erhöhte Infektionsanfälligkeit und weitere Verschlechterung des Allgemeinzustandes können die Folge sein. Das Bronchialsekret dieser Patienten verändert im Verlauf der Erkrankung durch verschiedene 
Faktoren seine Viskosität und wird durch mangelnde Mobilisation zäh und fest. Zusätzlich ist durch Tonusveränderungen der Rumpf- und Bauchmuskulatur das Abhusten erschwert.

\section{Sekretolyse - Mukoziliäre Clearance}

In der neurologischen Frührehabilitation haben viele Patienten neben ihrer neurologischen Grunderkrankung ein hohes bronchiales Sekretaufkommen mit massiv eingeschränkter mukoziliärer Clearance und erhöhter Infektanfälligkeit. Bei bronchitischen Infekten kommt es vorübergehend zu einer Lähmung, teilweise sogar zur Zerstörung des Flimmerepithels. Zudem führen Speichel- und Sekretaspirationen und chronische Bakterien- und Pilzkolonisation im Bronchialsystem zu erhöhten Pneumonieraten. Dabei nimmt die Häufigkeit des spontanen Hustens zu, je stärker die mukoziliäre Clearance eingeschränkt ist [3]. Bei invasiv beatmeten Patienten ist die mukoziliäre Clearance nahezu vollständig aufgehoben.

Das vermehrte und durch lange Immobilität zunehmend zähe Bronchialsekret kann von den Flimmerhärchen nur unzureichend bewegt werden und verbleibt oft in den tiefen Atemwegen. Ein Mangel an Surfactant erschwert zusätzlich die Ablösung und die Beförderung der zähen Schleimplaques [4]. Bei Tracheotomierten wird eine Hypersekretion durch die Reizung der Bronchialschleimhaut bei wiederholtem und vor allem bei unsachgemäßem Absaugen getriggert [5].

\section{Husten}

Voraussetzung für effektives Husten ist ein ausreichendes Atemvolumen, ein ausreichender subglottischer Druck und eine suffizient arbeitende, an der Atmung beteiligte Muskulatur. Bei Erkrankungen, bei denen eine Hypersekretion vorliegt, ist Husten der Hauptclearance-Mechanismus [6].

Dieser Mechanismus ist bei neurologischen Patienten zusätzlich aufgrund vorherrschender Muskelschwäche, durch Bettlägerigkeit und Lähmungen erschwert oder aufgehoben. Außerdem ist nicht selten die Koordination des Hustens gestört. Es kommt vermehrt zur Ausbildung von Dys- und Atelektasen, eingeschränkter Sauerstoffaufnahme und Atemwegsinfekten, die wiederum die respiratorische Dekompensation triggern [5].

Der tracheotomierte Patient zeigt ein niedriges Atemzugvolumen und einen insuffizienten intrapulmonalen Druckaufbau für effektives Husten. Zudem verhindert die Trachealkanüle den subglottischen Verschluss. Eine Hustenclearance ist nach Tracheotomie somit nahezu unmöglich, die Folgen sind Sekretverhalt mit erhöhter Pneumoniegefahr und Abnahme der Gasaustauschfläche. Letztlich wird häufig eine erneute Respiratortherapie notwendig, was eine vollständige Aufhebung der mukoziliären Clearance bedeutet.

\section{Konventionelle Atemtherapie und Sekretolyse in der Physiotherapie}

In der konventionellen physiotherapeutischen Atemtherapie kommen sehr unterschiedliche Ansätze und Hilfsmittel zur Unterstützung der Sekretclearance zur Anwendung. Allen gemeinsam ist, dass jede Eigenaktivität des Patienten einer passiven Maßnahme vorzuziehen ist. Wache und mobile Patienten können unterschiedliche Techniken und Lagerungen zur Sekretolyse und als Abhusthilfe erlernen, wie z. B. das Sniffing (schnüffelndes Einatmen) mit anschließendem Anhalten der Luft. Techniken, die eine forcierte Exspiration und Hustenunterstützung beinhalten, werden als effektivste Mittel der Physiotherapie zur Unterstützung des Sekrettransportes eingeschätzt [7].
Zur Hustenunterstützung soll der Patient seine Arme vor der Brust verschränken und so die notwendige Druckunterstützung geben. Verstärkend sollte dieses durch eine aktive Oberkörpervorlage ergänzt werden $[8,9]$. Einige Hilfsmittel, wie z.B. RC- Cornet $^{\circledR}$, VRP- Flutter ${ }^{\circledR}$ oder Acapella ${ }^{\circledR}$ choice, erzeugen Schwingungen im Exspirationsstrom und können zur Unterstützung der Sekretolyse verwendet werden. Eine weitere Möglichkeit ist die Applikation feuchter Wärme, die der Patient eigenverantwortlich anwendet. Auch kann dem Patienten ein Vibrationsgerät zur Verfügung gestellt werden, oder er kann sich bei festsitzendem Sekret selbst die Brust abklopfen.

Geiseler \& Karg (2008) [5] nennen verschiedene Techniken und Hilfsmittel zur Unterstützung des Sekretmanagements bei Patienten mit neuromuskulären Erkrankungen, darunter manuell assistiertes Husten, spezielle aktive Atemtechniken, maschinell unterstütztes Husten (Cough Assist ${ }^{\mathrm{TM}}$ ) und Air Stacking. Sie betonen die Wichtigkeit einer Differenzierung zwischen Maßnahmen zur Sekretmobilisierung und zur Sekretexpektoration und berichten über positive klinische Erfahrungen in der Anwendung dieser Maßnahmen. Bisher liegt jedoch nur wenig empirische Evidenz für deren Wirksamkeit vor.

Empirische Nachweise, dass konventionelle „Drainagelagerungen" und Vibrationstechniken einen positiven Effekt auf den Sekrettransport haben, stehen ebenfalls noch aus (vgl. [10-12]). Zwar wurden in einigen Studien positive Effekte auf die pulmonale Funktion und Blutgaswerte gefunden [13,14], diese Effekte könnten jedoch auch auf das durch die Lagewechsel verbesserte Ventilations-/Perfusionsverhältnis zurückführbar sein [7]. Für manuelle Techniken zur Hustenunterstützung konnte dagegen ein Sekretolyseeffekt gezeigt werden $[12,15]$.

\section{Besonderheiten bei Patienten in der neurologischen}

Frührehabilitation

Viele dieser atemtherapeutischen Ansätze sind, abgesehen von den regulären Lagerungswechseln zur Verbesserung des Ventilations-/Perfusionsverhältnisses, bei schwerstbetroffenen neurologischen Patienten nicht anwendbar, da sie die erforderliche Eigenaktivität nicht leisten können. Als Konsequenz ist es notwendig, eine erweiterte Atemtherapie in die Behandlung dieser Patienten einzubinden. Diese sollte über die bisher etablierten Ansätze wie Vibrationsmassagen, Abklopfen, Drainagelagerungen und medikamentöse Sekretolyse hinausgehen. Zwar kann durch herkömmliche Therapien das Sekret möglicherweise mobilisiert werden, aber die notwendige Expektoration des mobilisierten Sekretes ist dem Patienten in der Regel entweder gar nicht oder nur ineffektiv möglich. Das Sekret verbleibt also in den meisten Fällen in den unteren Atemwegen oder muss durch tiefes, „blindes“ Absaugen mit fatalen Konsequenzen (Triggerung einer Hypersekretion und Hyperplasie der sekretbildenden Drüsen) für das Bronchialsystem entfernt werden.

Die Methode des „Bagging“ und „Air Stacking“ wurde in der Aatalklinik Bad Wünnenberg als eine Möglichkeit für ein erweitertes Atemtherapiekonzept für Patienten der neurologischen Frührehabilitation modifiziert und in einer 6-monatigen Pilotphase erprobt. Ziel des Projektes war, die Durchführbarkeit der Methode und ihre Effekte hinsichtlich der Atemfunktion und Sekretolyse bei Patienten der Frührehabilitation in der Neurologie zu überprüfen. Im Folgenden werden das therapeutische Vorgehen beim „Bagging“ und „Air Stacking“ in Methodik und Anwendung erläutert sowie einige Evaluationsdaten zur Anwendung des Ansatzes vorgestellt. 


\section{Durchführung des „Bagging“ und „Air Stacking“ Bagging}

Bei der Methode des „Bagging“ (Bag = Beutel) wird dem Patienten mit Hilfe eines herkömmlichen Beatmungsbeutels atemsynchron während der Inspiration zusätzlich Luft in die Atemwege insuffliert. Wichtig hierbei ist, dass der Patient zunächst selbst die Inspiration einleitet und die Insufflation dann direkt auf die Inspiration „aufgesetzt“ wird. Durch die entstehende Atemvolumenerhöhung werden minderbelüftete Areale und Dys- und Atelektasen rekrutiert, die dann am Gasaustausch wieder teilnehmen und den Rechts-Links-Shunt vermindern können. Durch die Aktivierung zusätzlich belüfteter Areale wird das Sauerstoffangebot erhöht und die Kontaktzeit in der Alveole verlängert. Auch kann sich die insufflierte Luft distal des Sekrets platzieren und ist so in der Lage, während der Exspiration den Sekrettransport oralwärts zu unterstützen. Nach einigen Bagging-Intervallen, deren Länge bzw. Häufigkeit individuell an den Patienten anzupassen ist, erfolgt eine verbale Aufforderung zum aktiven Abhusten des so mobilisierten Sekretes. Da die Exspirationskraft der Patienten besonders bei zähem Sekret für ein produktives Husten in der Regel nicht ausreicht, wird von einer Hilfsperson eine manuelle Hustenunterstützung durchgeführt (vgl. @ Abb.1). Hierzu kann wahlweise durch bimanuelles seitliches Zusammendrücken der Rippen oder durch Druck unterhalb des epigastrischen Winkels von vorn der intrathorakale Druck erhöht werden $[10,16]$. Durch dieses unterstützte Hustenmanöver erhöhen sich die Kraft des Hustenstoßes und die Effektivität der Expektoration.

Die beschriebene Methode kann unabhängig von der Vigilanz des Patienten angewendet werden. Die Anzahl der Insufflationen ist an den aktuellen Atemrhythmus und den individuellen Gesundheitszustand des Patienten anzupassen. Bei wacheren Patienten, die in der Lage sind, dem Ablauf zu folgen und ihn aktiv mitzugestalten, kommen unterschiedliche Steigerungsmöglichkeiten des Baggings in Betracht. Nach einer Inspiration wird der Patient aufgefordert, die Einatmung maximal zu vertiefen und am Ende der Inspirationsphase die Luft anzuhalten, bevor die Exspiration eingeleitet wird. Die eingeatmete Luft hat so mehr Zeit sich in den Alveolen zu verteilen bzw. sich hinter das Sekret zu setzen. Eine weitere Steigerung beinhaltet, direkt nach der maximalen Inspiration mit Einbehalten der Luft sofort kräftig auszuhusten. Dies wird zur Erleichterung und Verstärkung manuell durch den Therapeuten unterstützt.

Während bei nicht-tracheotomierten Patienten die Insufflationen über eine Beatmungsmaske gegeben werden, erfolgt das Bagging bei tracheotomierten Patienten durch Ansetzen des Beatmungsbeutels auf das Kanülenende (vgl. $\bigcirc$ Abb.2). Es empfiehlt sich die Verwendung eines Bakterienfilters. Die Anwendung ist im Folgenden bei beiden Patientengruppen gleich. Selbst wenn es trotz der manuellen Hustenunterstützung nicht zum Sekrettransport aus der Trachealkanüle kommt, reicht sie doch häufig aus, um das Sekret in den Schaft der Trachealkanüle zu transportieren, so dass der Patient lediglich innerhalb der Kanüle abgesaugt werden muss. Dies bedeutet einen erheblich verminderten Reiz und eine Minimierung der Schleimhautverletzungen an der Trachea. Bei Patienten mit einer geblockten Trachealkanüle ist die Möglichkeit des Luftanhaltens als Steigerungsoption jedoch nicht gegeben, da kein subglottischer Druck aufgebaut werden kann. Hier kann durch Verwendung eines Einwegventils zwischen Bakterienfilter und Beatmungsbeutel ein passiver tracheobronchialer Staudruck erzielt werden.

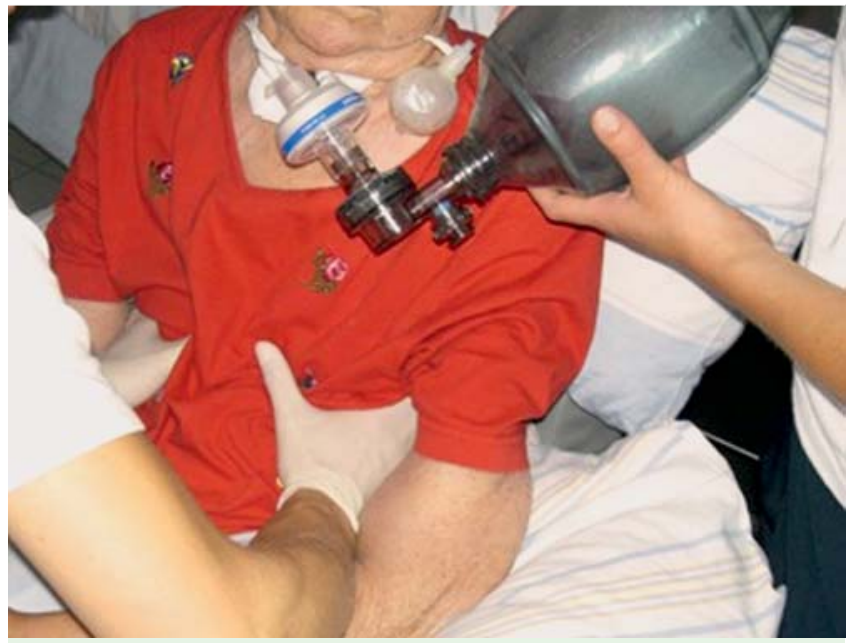

Abb. 1 Manuelle Hustenunterstützung an der unteren Thoraxapertur mit Druck nach medial und cranial. Das Sekret wird so unterstützend kanülenwärts transportiert.

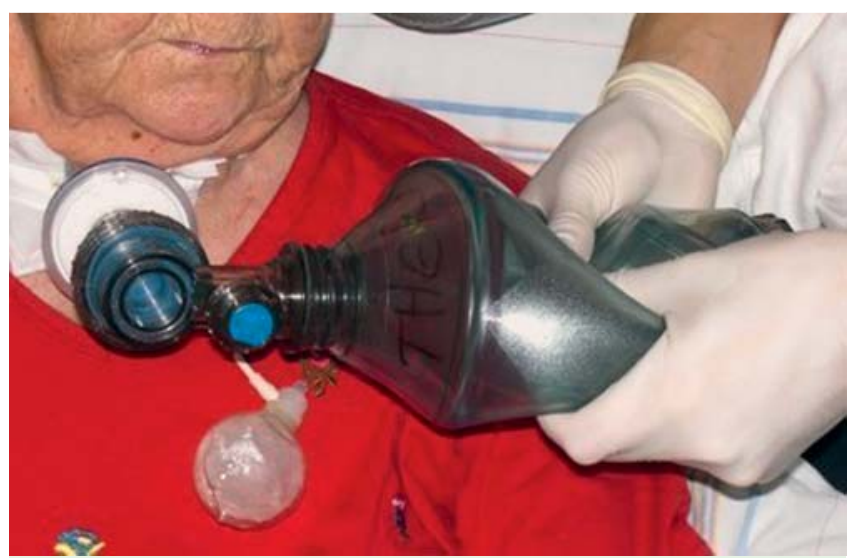

Abb. 2 Bagging: Unterstützung der Inspiration durch manuelle Hyperinsufflation mit Hilfe eines Beatmungsbeutels bei einer Patientin mit geblockter Trachealkanüle.

\section{Kontraindikationen}

Bei Z.n. abdominalen Operationen bzw. Thorakotomien, Wirbelund Rippenserienfrakturen, Knochenmetastasierung und bei schwerer Osteoporose ist die Anwendung des Bagging und Air Stacking kontraindiziert. Chronisch obstruktive Lungenerkrankungen (z. B. COPD, Asthma, Emphysem) bilden relative Kontraindikationen, hier ist die Anwendung des Baggings individuell und unter sorgfältiger Analyse von Nutzen und Risiken zu entscheiden. Die Bagging-Anwendung sollte nicht unmittelbar nach der Nahrungsaufnahme erfolgen, da durch den Druck auf das Epigastrium während der manuellen Hustenunterstützung die Gefahr von Reflux und Erbrechen und hierdurch ein erhöhtes Aspirationsrisiko besteht.

\section{Air Stacking}

Eine weitere Steigerungsmöglichkeit bildet sowohl bei tracheotomierten als auch bei nicht-tracheotomierten Patienten das „Air Stacking“. Hier werden mehrere Inspirationen mit dem Beatmungsbeutel unterstützt, ohne dass zwischenzeitlich eine Exspiration erfolgt. Die aufeinander „gestapelten“ Inspirationen dienen der maximalen Blähung des Lungengewebes. Man beginnt mit wenigen „gestackten“ Inspirationen, bevor es zur manuellen 
Hustenunterstützung kommt. Die Steigerungen müssen individuell angepasst werden. Durch das Air Stacking wird der intrapulmonale Druck erhöht und der Hustenstoß kräftig und effektiv. Für Patienten mit neuromuskulären Erkrankungen konnten empirische Nachweise erbracht werden, dass durch eine Kombination von Air Stacking, manueller Hustenunterstützung und spezieller Atemtechnik die Husteneffektivität deutlich gesteigert werden kann [17,18] (vgl. auch [5]). Das Stacking ist umso effektiver, je vigilanter der Patient ist, da die aktiv gesteigerten Inspirationen deutlich tiefer sind als die aufgesetzten. Außerdem kommt es zu einer physiologischen, gleichmäßigen Verteilung der Luft. Bei vigilanzgeminderten und tracheotomierten Patienten wird mit Hilfe eines Einwegventils zwischen Bakterienfilter und Beatmungsbeutel das zwischenzeitliche Ausatmen verhindert. Hier werden nicht mehr als drei Inspirationshübe aufeinander gesetzt. Der Beobachter sieht dabei eine deutliche Hebung des Brustkorbs und ein Anheben des Schultergürtels.

\section{Material und Methoden}

\section{$\nabla$}

\section{Fragestellung}

Bagging und Air Stacking sind Methoden zur Sekretolyse und Bronchialtoilette, die über die bisher bekannten physiotherapeutischen Maßnahmen hinausgehen und auch bei schwerst betroffenen und tracheotomierten Patienten anwendbar sind. Zur Evaluation dieser Methoden wurde überprüft, ob die Anwendung des Bagging und Air Stacking bei neurologischen Patienten der Phase $\mathrm{B} \mathrm{zu}$ verbesserten $\mathrm{SPO}_{2}$-Werten führt. Dies wäre ein Hinweis darauf, dass eine Verbesserung der Sekretolyse und des Sekrettransportes und somit eine Erhöhung der Gasaustauschfläche durch die Bagging-Technik erreicht werden konnte (vgl. $[5,18]$ ). Als Evaluationszeitraum wurde dabei ein Behandlungszeitraum von 12 Tagen festgelegt.

\section{Probanden}

Zielgruppe des Projektes waren vor allem tracheotomierte Patienten, ggf. auch unter Respiratortherapie, mit unzureichender Sekretclearance. Ausschlusskriterien waren eine vorbekannte COPD, Z.n. Pleuradrainage oder Thorakotomien sowie Z.n. Rippenserienfrakturen oder hochgradige Osteoporose.

Während der Evaluationsphase wurden 11 Patienten behandelt $(\mathrm{w}=2, \mathrm{~m}=9)$ im durchschnittlichen Alter von 67 (21 - 74) Jahren. Zehn dieser Patienten waren tracheotomiert (Primärdiagnosen: hypoxischer Hirnschaden $n=5$ und Schädel-Hirn-Trauma Grad III $n=5$ ), ein Patient wurde zeitweise druckunterstützt beatmet. Eine Patientin war nicht tracheotomiert, sie wurde aufgrund der Primärdiagnose Multiple Sklerose mit einer schweren Tetraspastik behandelt. Alle Patienten waren zuvor im Akutkrankenhaus mindestens einmal antibiotisch aufgrund einer Pneumonie behandelt worden und hatten ein vermehrtes bronchiales Sekretaufkommen sowie eine verminderte Husteneffektivität. Im röntgenologischen Aufnahmebefund konnten pneumonische Infiltrate, Dys- und/oder Atelektasen nachgewiesen werden.

\section{Durchführung und Dokumentation der Therapie}

In der evaluierten Therapiephase wurde das Bagging bei jedem Patienten 1-2 $\times$ täglich, wie oben beschrieben, über zwölf Werktage durchgeführt. Der Patient wurde in eine angemessene Ausgangsstellung (Sitz oder Seitenlage) gebracht. Um den Patienten selbst und die verkürzten thorakalen Strukturen an das vermehrte Luftangebot zu gewöhnen, wurden zunächst 5-10 Insufflatio-
Tab. 1 Dokumentationsbogen Pilotprojekt „Bagging“, Aatalklinik Wünnenberg.

\begin{tabular}{|c|c|}
\hline Parameter & Erläuterung \\
\hline \multicolumn{2}{|l|}{ 1. Datum, Zeit } \\
\hline \multicolumn{2}{|l|}{ 2. Ausgangsstellung/Lagerung } \\
\hline 3. $\mathrm{SPO}_{2}$-Messung & $\begin{array}{l}\text { bei allen Probanden vor und nach } \\
\text { jeder Bagging-Anwendung }\end{array}$ \\
\hline $\begin{array}{l}\text { 4. Peak- Flow-Messung/ } \\
\text { Spirometertest }\end{array}$ & $\begin{array}{l}\text { bei nicht-tracheotomierten Proban- } \\
\text { den, sofern } \mathrm{SPO}_{2} \text {-Wert unauffällig }\end{array}$ \\
\hline 5. Frequenz/Serie & $\begin{array}{l}\text { Anzahl und Wiederholungszahl der } \\
\text { Insufflationen }\end{array}$ \\
\hline $\begin{array}{l}\text { 6. Exspiratorischer manueller } \\
\text { Druck } \\
\text { (produktiv/unproduktiv) }\end{array}$ & $\begin{array}{l}\text { produktiv = Expektoration bis in den } \\
\text { Kanülenschaft möglich } \\
\text { unproduktiv = keine oder unzurei- } \\
\text { chende Expektoration }\end{array}$ \\
\hline 7. Absaugfrequenz & $\begin{array}{l}\text { Anzahl der notwendigen Absaugun- } \\
\text { gen vor, während und nach der Maß- } \\
\text { nahme }\end{array}$ \\
\hline
\end{tabular}

nen mit dem Beatmungsbeutel vorgenommen, bevor eine manuelle Hustenunterstützung erfolgte. Zeigte der Patient eine ausgeprägte Tachypnoe, wurde lediglich jeder 2.-3. Atemzug gebaggt. So konnte sich der Therapeut besser in den Rhythmus des Patienten einfinden. Oft änderte das ungewohnte, zusätzliche Luftangebot auch reflektorisch den Atemrhythmus des Patienten und seine Atmung verlangsamte sich.

Für die individuelle Verlaufsdokumentation wurde ein Dokumentationsbogen entwickelt (vgl. $\bullet$ Tab. 1).

\section{Ergebnisse \\ $\nabla$}

\section{Verlauf der $\mathrm{SPO}_{2}$-Werte im Behandlungszeitraum}

In $\triangle$ Abb. 3 werden die Messergebnisse im Gruppenmittel vor und nach dem Bagging dargestellt. Die Anwendung des Bagging und Air Stacking führte bei den Patienten an jedem Behandlungstag zu einer deutlichen Erhöhung des $\mathrm{SPO}_{2}$-Wertes. So konnte bereits am ersten Behandlungstag eine Verbesserung im Gruppenmittel von $90 \% \mathrm{SPO}_{2}\left(87\right.$ - 93) auf 94,7\% $\mathrm{SPO}_{2}(92-98)$ erreicht werden. Auch über die gesamte zwölftägige Therapiephase hinweg kam es zu einem kontinuierlichen Anstieg der $\mathrm{SPO}_{2}$-Werte der behandelten Patienten. Dies wird einerseits deutlich am Verlauf der $\mathrm{SPO}_{2}$-Werte nach jeder Anwendung als auch in der Beobachtung, dass auch die Ausgangswerte der Patientengruppe an nahezu jedem Behandlungstag höher waren als am vorangegangenen Tag. So verbesserte sich die gesamte Gruppe bezogen auf die Ausgangswerte von $90 \% \mathrm{SPO}_{2}(87-93)$ zu Beginn der Therapiephase auf durchschnittlich 95,3\% $\mathrm{SPO}_{2}(94-98)$ nach Ende der Therapiephase. Durch die Anwendung der Methode konnte somit nicht nur ein temporärer Anstieg der Sauerstoffsättigung am Behandlungstag erreicht werden. Dieser positive Effekt war stabil und somit nachhaltig.

Die Ergebnisse wurden in einer Einzelfallanalyse für alle behandelten Patienten weiter überprüft. Hierzu wurden 3 Kategorien von $\mathrm{SPO}_{2}$-Werten gebildet, wobei die Ausgangs- und Endwerte aller Patienten zwischen dem niedrigsten (87\%) und höchsten Wert (98\%) gleichmäßig aufgeteilt folgende Einteilung ergaben: In die erste Kategorie fielen $\mathrm{SPO}_{2}$-Werte (vor Bagging) von $\leq 90 \%$, in die zweite Kategorie fielen Werte von $\leq 94 \%$ und in die dritte Kategorie Werte von $>94 \% \mathrm{SPO}_{2}$ (vgl. $\odot$ Tab. 2 ). 


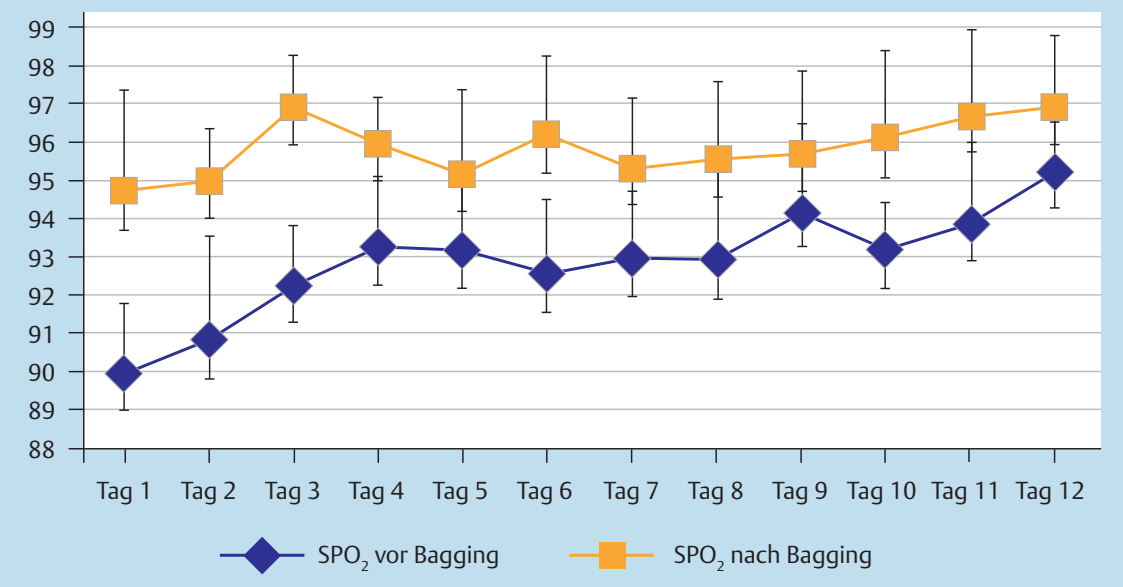

Abb. 3 Verlauf der $\mathrm{SPO}_{2}$ - Sättigung vor und nach Anwendung des Bagging. Gruppenmittelwerte über 12 Behandlungstage.

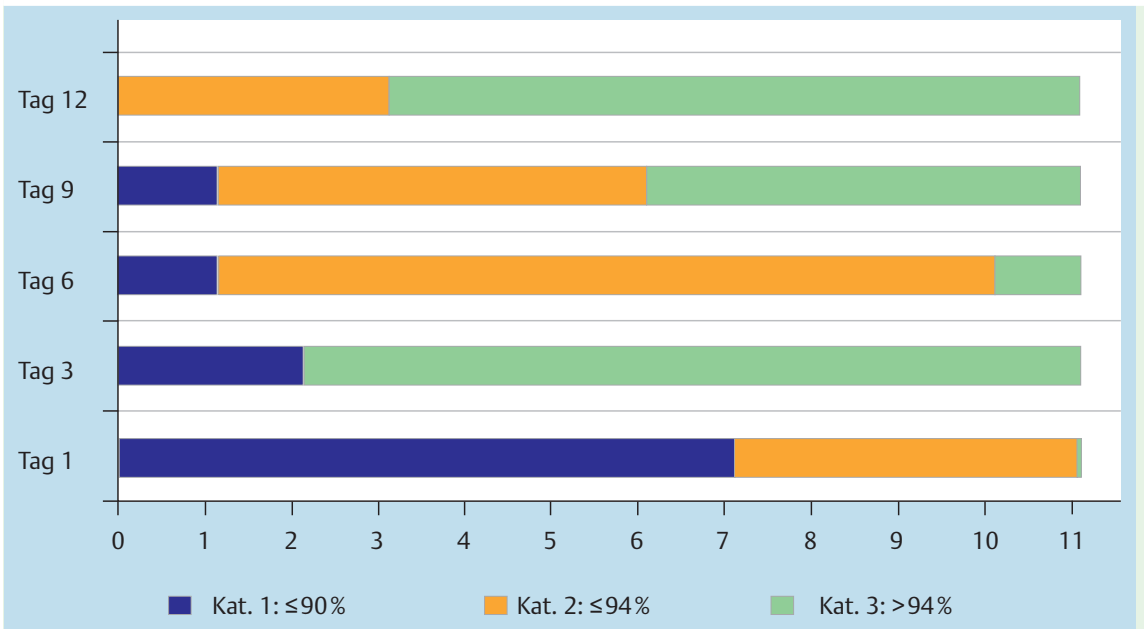

Abb. 4 Verlauf der $\mathrm{SPO}_{2}$ - Ausgangswerte bei 11 Patienten vor Anwendung des Bagging. Einteilung der Werte in 3 Kategorien und Darstellung der Patientenanzahl in jeder Kategorie an 5 Behandlungstagen (3-Tages-Intervall).

Die Ergebnisse dieser Einzelfallanalyse sind in $\bullet$ Abb. 4 dargestellt. Alle Patienten wurden zu Beginn der Therapiephase (Tag $1)$ entweder der Kategorie $1(n=7)$ oder der Kategorie $2(n=4)$ zugeordnet, kein Patient erreichte Kategorie 3 (entsprechend $>94 \% \mathrm{SPO}_{2}$ ). Dagegen kam es im Behandlungsverlauf für alle Patienten zu einem „Aufstieg“ in eine höhere Kategorie. So war z. B. bereits am 6. Behandlungstag nur noch 1 Patient der Kategorie $1(\leq 90 \%)$ zuzuordnen, 9 Patienten konnten mit einem $\mathrm{SPO}_{2}-$ Wert von $\leq 94 \%$ der Kategorie 2 zugeordnet werden und ein Patient wies bereits einen Wert von $>94 \%$ auf (Kategorie 3). Am Ende der Therapiephase am Tag 12 war kein Patient mehr der Kategorie 1 zuzuordnen, dagegen 3 Patienten der Kategorie 2 ( $\leq 94 \%$ ) und 8 Patienten der Kategorie 3 (>94\%). Bei keinem Patienten kam es zu Verschlechterungen bzw. zu einem „Rückfall“ in eine niedrigere Kategorie.

Auch die Einzelfallanalyse bestätigt somit die Beobachtung, dass durch die Anwendung des Bagging eine stabile und nachhaltige Verbesserung des $\mathrm{SPO}_{2}$ für jeden einzelnen behandelten Patienten erzielt werden konnte. Ein Zusammenhang zwischen Therapieverlauf und Diagnose der individuellen Patienten konnte dabei nicht hergestellt werden.

\section{Explorative Beobachtung weiterer klinischer Effekte}

Es zeigten sich während der Gesamtlaufzeit des Projektes (über 6 Monate) noch eine Reihe weiterer Nebeneffekte. Diese Beobachtungen wurden jedoch nicht systematisch evaluiert, daher erfolgt hier eine deskriptive Beschreibung. Auffällig war, dass es bei apallischen Patienten manchmal während der ersten Anwendun-
Tab. 2 Kategorisierung der $\mathrm{SPO}_{2}$-Einzelwerte, relevant sind hier die täglichen Werte vor Anwendung der ersten Bagging-Behandlung.

$\begin{array}{ll}\text { Kategorie } 1 & \leq 90 \% \mathrm{SPO}_{2} \\ \text { Kategorie 2 } & \leq 94 \% \mathrm{SPO}_{2} \\ \text { Kategorie 3 } & >94 \% \mathrm{SPO}_{2}\end{array}$

gen zu Apnoephasen kam. Evtl. könnte dies durch eine Hemmung des zentralen Atemantriebs bedingt sein, die durch das plötzliche vermehrte Luftangebot ausgelöst wird. Vermutlich spielen auch die Dehnungsrezeptoren der Atemmuskulatur eine Rolle, die eine drohende Überdehnung der Muskelspindeln zum zentralen Nervensystem senden. Wenn diese Apnoephasen über 30 Sekunden anhielten, wurde durch das oben beschriebene manuelle Handling am Thorax die Luft ruckartig in 2-3 Schritten „ausgepumpt“.

Als positiver Effekt zeigte sich bei den Patienten, die regelmäßig eine Bagging-Anwendung erhielten, eine auffällig rasche Zunahme der Vigilanz. Zudem veränderte sich die Viskosität des Sekrets positiv (von fest über zäh zu flüssig), sodass die Patienten deutlich leichter abhusten konnten. Die Infektanfälligkeit schien deutlich vermindert, beginnende Infekte mit Temperaturanstieg wurden bei gleicher Medikation schneller überwunden. Bei allen in das Projekt integrierten Patienten verbesserte sich der Allgemeinzustand. Von den Logopäden wurden schnellere Erfolge in der Dysphagietherapie beobachtet. Daraus resultierte häufig eine raschere Um- und/ oder Dekanülierung der tracheotomierten Patienten. Außerdem fiel eine kräftigere Stimmgebung bei 
gebaggten Patienten auf und zwar sowohl mit entblockter Trachealkanüle in situ und Sprechaufsatz als auch nach der Dekanülierung.

Zusammenfassend legen diese Beobachtungen den Schluss nahe, dass die Anwendung des Bagging und Air Stacking über den objektiv messbaren Parameter der $\mathrm{SPO}_{2}$-Werte hinaus zu positiven Effekten im Rehabilitationsverlauf führt. Eine systematische Studie zur Untersuchung dieses postulierten Zusammenhangs ist derzeit in Planung.

\section{Diskussion}

Bei Patienten in der neurologischen Frührehabilitation kommt es neben den Symptomen der neurologischen Primärdiagnose immer häufiger zu pneumonologischen Begleitkomplikationen. Bei diesen Patienten können jedoch nur wenige Therapieansätze und Hilfsmittel zur Sekretolyse und Sekretexpektoration angewendet werden. Ein gutes Sekretmanagement bildet die Grundvoraussetzung für die weiterführende Mobilisation des Patienten und somit auch für weitere Fortschritte in der Rehabilitation, da durch den verbesserten Gasaustausch eine Kreislaufstabilisation, eine Verminderung kataboler Zustände und letztlich häufig auch eine Vigilanzsteigerung erreicht werden kann. Dies wirkt sich auch positiv auf die Rehabilitation funktioneller Aktivitäten aus. Herkömmliche physiotherapeutische und physikalische Maßnahmen sind bei schwer betroffenen Patienten unterstützend anwendbar, sie sind jedoch für eine effektive Bronchialtoilette keinesfalls ausreichend.

Die hier vorgestellten ersten Evaluationsdaten zeigten, dass die Bagging-Methode bei den untersuchten Probanden zu einer stabilen und nachhaltigen Verbesserung der peripheren $\mathrm{O}_{2}$-Sättigung führte und somit ein erfolgversprechender Ansatz zur Sekretolyse und Sekretexpektoration bei neurologischen Patienten in der Frührehabilitation ist. Neben dem messbaren atemphysiologischen Effekt konnten auch positive Effekte bei weiteren klinischen Parametern beobachtet werden, wie das Infektions- und Sekretmanagement, aber auch auf weitere, mit der Atmung verbundene Funktionen, wie z.B. die Schluckfunktion und die Stimmgebung. Die Methode ist darüber hinaus leicht zu erlernen und die Materialkosten sind gering, sodass sie leicht in die klinisch-therapeutischen Abläufe zu integrieren ist.

Aufgrund der Projektergebnisse wurde das Bagging und Air Stacking in der Aatalklinik Wünnenberg bereits in das atemtherapeutische Konzept implementiert, und es wird derzeit weiterentwickelt. Im Rahmen eines interdisziplinären Ansatzes werden physikalische Wärmeanwendungen (heiße Rolle) zeitlich direkt vor der Maßnahme durchgeführt, um so eine effektivere Vorbereitung der Muskulatur und der Sekretverflüssigung zu erreichen. Darüber hinaus erfolgt eine Schulung und Integration weiterer therapeutischer Berufsgruppen und des Pflegepersonals, damit die Maßnahmen unabhängig von der Personalzusammensetzung konsequent und mehrmals täglich zur Anwendung kommen können.
Weitere Studien sind notwendig, um diese ersten Ergebnisse mit größeren Patientengruppen und auch mit spezifischen Diagnosegruppen abzusichern und Erkenntnisse zur Optimierung des beschriebenen Verfahrens zu erlangen. Dabei sollte insbesondere systematisch untersucht werden, ob und wie sich das Bagging und Air Stacking positiv auf weitere mit der Atmung relatierte Funktionen, wie z.B. die Schluckfunktion und die Phonation, auswirken kann.

\section{Interessenkonflikte}

$\nabla$

Die Autoren geben an, dass kein Interessenkonflikt besteht.

\section{Literatur}

1 Seeley HM, Hutchinson PJ. Rehabilitation following traumatic brain injury: challenges and opportunities. ACNR 2006; 6: 22-28

2 Schönle PW, Stemmer B. Neurologische Rehabilitation in den Phasen B C, D und E. Praxis und Prognose. Bad Honnef: Hippocampus; 2000

3 Köhler D. Physiologie und Pathophysiologie des Hustens. Pneumologie 2008; 62: $14-17$

4 Edel H, Knauth K. Atemtherapie. München: Urban \& Fischer; 1999

5 Geiseler J, Karg O. Sekretmanagement bei neuromuskulären Erkrankungen. Pneumologie 2008; 62 (1): $43-48$

6 Mossberg B, Camner P. Mucociliary transport and cough as tracheobronchial clearance mechanisms in pathological conditions. Europ J Respir Dis 1980; 61 Suppl 110: 47 - 55

7 Van der Schans CP, Postma DS, Koeter GH et al. Physiotherapy and bronchial mucus transport. Eur Respir J 1999; 13: 1477-1486

8 Ehrenberg $H$. Atemtherapie in der Physiotherapie. München: Pflaum Krankengymnastik; 2001

9 Van Gestel AJR, Teschler H. Physiotherapie bei chronischen Atemwegsund Lungenerkrankungen. Berlin: Springer; 2010

10 Bach JR. Mechanical insufflation-exsufflation. Comparison of peak expiratory flows with manually assisted and unassised coughing techniques. Chest 1993; 104: 1553-1562

11 Eid N, Buchheit J, Neuling M, Phelps $H$. Chest physiotherapy in review. Respir Care 1991; 36: 270-282

12 Bateman JRM, Newman SP, Daunt KM et al. Is cough as effective as chest physiotherapy in the removal of excessive tracheobronchial secretions? Thorax 1981; 36: 683-687

13 Dhainaut JF, Bons J, Bricard C et al. Improved oxygenation in patients with extensive unilateral pneumonia using the lateral decubitus position. Thorax 1980; 35: $792-793$

14 Gillespie DJ, Rehder K. Body position and ventilation-perfusion relationships in unilateral pulmonary disease. Chest 1987; 91: $75-79$

15 de Boeck C, Zinman R. Cough vs. chest physiotherapy: a comparison of the acute effects on pulmonary functions in patients with cystic fibrosis. Am Rev Respir Dis 1984; 129: 182 - 184

16 Massery $M$, Frownfelter $D$. Assisted cough techniques - there's more than one way to cough. Phys Ther Forum 1990; 9: 1-4

17 Feigelson CI, Dickinson DG, Talner NS et al. Glossopharyngeal breathing as an aid to the coughing mechanism in the patient with chronic poliomylitis in a respirator. N Engl J Med 1956; 254: 611-613

18 Kirby NA, Barnerias MJ, Siebens AA. An evaluation of assisted cough in quadriplegic patients. Arch Phys Med Rehabil 1966; 47: 705-710

19 Bach JR. The Oximetry Feedback Respiratory Aid Protocol. In: Bach JR Management of patients with neuromuscular disease. Philadelphia: Hanley \& Belfus; 2004 